\title{
The magnitudes and timescales of global mean surface temperature feedbacks in climate models
}

\author{
A. Jarvis \\ Lancaster Environment Centre, Lancaster University, Lancaster, LA1 4YQ, UK \\ Received: 28 May 2011 - Published in Earth Syst. Dynam. Discuss.: 1 July 2011 \\ Revised: 22 November 2011 - Accepted: 1 December 2011 - Published: 15 December 2011
}

\begin{abstract}
Because of the fundamental role feedbacks play in determining the response of surface temperature to perturbations in radiative forcing, it is important we understand the dynamic characteristics of these feedbacks. Rather than attribute the aggregate surface temperature feedback to particular physical processes, this paper adopts a linear systems approach to investigate the partitioning with respect to the timescale of the feedbacks regulating global mean surface temperature in climate models. The analysis reveals that there is a dominant net negative feedback realised on an annual timescale and that this is partially attenuated by a spectrum of positive feedbacks with characteristic timescales in the range 10 to $1000 \mathrm{yr}$. This attenuation was composed of two discrete phases which are attributed to the equilibration of "diffusive - mixed layer" and "circulatory - deep ocean" ocean heat uptake. The diffusive equilibration was associated with time constants on the decadal timescale and accounted for approximately 75 to 80 percent of the overall ocean heat feedback, whilst the circulatory equilibration operated on a centennial timescale and accounted for the remaining 20 to 25 percent of the response. This suggests that the dynamics of the transient ocean heat uptake feedback first discussed by Baker and Roe (2009) tends to be dominated by loss of diffusive heat uptake in climate models, rather than circulatory deep ocean heat equilibration.
\end{abstract}

Correspondence to: A. Jarvis (a.jarvis@lancs.ac.uk)

\section{Introduction}

Resolving global climate feedbacks has been an important feature of climate change research for many years (Hansen et al., 1984, 1985; Bony et al., 2006). Past research has tended to focus on either instantaneous or equilibrium timescale climate feedbacks given these are amenable to steady state analysis (e.g. Colman 2003; Gregory et al., 2004; Soden and Held, 2006; Bony et al., 2006; Forster and Taylor, 2006; Roe and Baker, 2007; Gregory and Forster, 2008; Lu and Cai, 2009). Although both of these timescales are clearly important, feedbacks in the climate system operate over a broad spectrum of timescales ranging from days to millennia (Hansen et al., 1985), and it is important that we characterise these also (Hansen et al., 2007; Knutti and Hegerl, 2008; Roe, 2009).

The timescales of climate feedbacks are determined by the dynamics of the climate system (e.g. Hoffert et al., 1980; Watts et al., 1994; Stouffer, 2004; Danabasoglu and Gent, 2009; Baker and Roe, 2009; Roe, 2009) with the atmosphere dominating the behaviour of the shorter timescale sub-annual feedbacks, and the oceans the longer timescale feedbacks (Hansen et al., 1985; Watts et al., 1994; Dickinson and Schaudt, 1998; Baker and Roe, 2009). Attributing feedbacks to specific timescales is more difficult than analysing the equilibrium condition because one has to account for the transient response of the climate system (e.g. Bates, 2007; Roe, 2009; Baker and Roe, 2009). Simple climate models have been the main vehicle for exploring transient feedback timescales (e.g. Hansen et al., 1985; Wigley and Schlesinger, 1985; Dickinson and Schaudt, 1998; Hallegatte et al., 2006; Bates, 2007). Of particular relevance here is the work of

Published by Copernicus Publications on behalf of the European Geosciences Union. 
Baker and Roe (2009) who investigated the effect of a transient oceanic heat uptake feedback on equilibrium climate sensitivity and transient climate change. Using a simple 1d upwelling-diffusion Global Energy Balance Model (GEBM) they show the importance of feedback timescale when inferring climate sensitivity. However, we are also interested in transient feedback analysis of more detailed climate system descriptions such as Earth system Models of Intermediate Complexity (EMICs) and Atmosphere-Ocean General Circulation Models (A-OGCMs). Because these models contain more complex dynamic mechanisms, numerical methods are required to characterise their transient feedback behaviour (Prather, 1996). Gregory and Forster (2008) and Williams et al. (2008) estimated the feedback strength associated with the transient response of global mean surface temperature in a range of A-OGCMs using the static linear analysis method of Gregory et al. (2004). An alternative approach would be to develop an explicitly dynamic framework for identifying feedback timescales directly.

It is important that feedback timescales are not simply seen as the time evolution of the strength of the feedback(s) being studied (see e.g. Williams et al., 2008; Baker and Roe, 2009), because this will be a function of both the dynamics of interest and the specific input used to excite this behaviour. A more appropriate approach would be to consider feedback strength in relation to standard measures of dynamic timescale such as time constants which, for a linear system, are fixed properties. Although there is good evidence to suggest that climate models exhibit many nonlinear traits (Aires and Rossow, 2003), particularly in their local distributed response, there is also evidence to show that the perturbed global aggregate response of climate models is often near linear (e.g. Li and Jarvis, 2009); an assumption that underpins most of the published work on climate feedbacks (e.g. Gregory et al., 2004; Bony et al., 2006; Forster and Taylor, 2006; Roe and Baker, 2007; Gregory and Forster, 2008).

In this paper we present a numerical methodology for estimating the strength (amplitude) of feedbacks operating on the surface temperature dynamics of climate models following $\mathrm{CO}_{2}$ induced perturbations in radiative forcing. Like much previous work on climate feedbacks the analysis will be based on the annual, global mean response. However, unlike previous studies that explicitly set out to differentiate surface temperature feedbacks with respect to climate processes, here the feedbacks will be differentiated with respect to the timescale (time constant) of their response to surface temperature change. This is important because it will allow an evaluation of the relative magnitudes of the fast and slow (and all intervening timescales) feedbacks operating in these models.

\subsection{Methods}

\subsubsection{Definition of climate reference and feedback systems}

Because feedback is the generation of reference system input from its output, feedbacks can only be defined in relation to the reference system on which they operate (Stephens, 2005; Roe, 2009). If $\Delta T(\mathrm{~K})$ are annual mean global surface temperature perturbations relative to a pre-industrial baseline; $Q$ $\left(\mathrm{W} \mathrm{m}^{-2}\right)$ are net annual mean global energy inputs to the surface independent of $\Delta T$ (which we will call the exogenous forcing); and $F\left(\mathrm{~W} \mathrm{~m}^{-2}\right)$ are net annual mean global net energy inputs to the surface which are dependent on $\Delta T$ (i.e. $F=f\{\Delta T\}$; which we will call the feedback forcing), then the global surface energy balance reference system can be described by,

$C \frac{\mathrm{d}}{\mathrm{d} t} \Delta T=Q+F$

where $C$ is the effective heat capacity of this element of the climate system. This heat capacity is predominately determined by the heat capacity of the well-mixed surface ocean, given the land and atmosphere, to which the surface ocean is strongly coupled through large exchanges of sensible and latent heat, have relatively little thermal inertia (Dickinson and Schaudt, 1998). In defining the global surface energy balance reference system in this way we assume it to be bounded by the top of the atmosphere, across which radiant heat is exchanged with space, and by the ocean thermocline, across which sensible heat is exchanged with the intermediate and deep ocean (Dickinson and Schaudt, 1998). We also assume it to be homogenous with respect to $\Delta T$, although to accommodate this assumption $c$ is not defined a priori but will instead be estimated from the transient climate model data.

Assuming linearity, the feedback forcing $F$ can be considered as the sum of $N$ feedbacks terms,

$F=\sum_{i=1}^{N} F_{i}$.

For each of these $N$ feedbacks we can specify a time constant, $\tau_{i}(\mathrm{yr})$, to represent the timescale over which the feedback equilibrates to a perturbation in $\Delta T$, and an amplitude, $g_{i}\left(\mathrm{~W} \mathrm{~m}^{-2} \mathrm{~K}^{-1}\right)$, to represent the contribution of the feedback to the equilibrium response of $F$ following a perturbation in $\Delta T$. Taking a linear systems approach, each individual feedback can now be considered as a first order subsystem (see Fig. 1) where,

$F_{i}=g_{i} \Delta T-\tau_{i} \frac{\mathrm{d}}{\mathrm{d} t} F_{i}$.

Because of the use of transient feedbacks in the climate literature (e.g. Williams et al., 2008; Baker and Roe, 2009) it is 
also useful to consider the amplitude of the cumulative feedback experienced at timescale $\tau, G(\tau)\left(\mathrm{W} \mathrm{m}^{-2} \mathrm{~K}^{-1}\right)$, which is given by,

$G(\tau)=\sum_{\tau=0}^{\tau} g(\tau)$

Providing the climate system is stable and fully damped, as $\tau \rightarrow \infty$ the closed loop system described by Eqs. (1 to 3 ) reduces to,

$\Delta T(\infty)=\frac{1}{-G(\infty)} Q(\infty)$

and hence the equilibrium climate sensitivity, $S(\mathrm{~K})$, is given by,

$S=\frac{1}{-G(\infty)} Q_{2 \times \mathrm{CO}_{2}}$

where $Q_{2 \mathrm{xCO}_{2}}$ is the exogenous forcing associated with a doubling in atmospheric $\mathrm{CO}_{2}$ from a pre-industrial baseline. Hence $G$ resembles the static feedback parameters and sensitivities commonly discussed in the climate literature (e.g. Forster and Taylor, 2006). However, it is important to appreciate that $G$ only corresponds to these more familiar climate feedback representation as $\tau \rightarrow \infty$ and the dynamics in the climate system have been fully extinguished. For all intermediate timescales the feedback sensitivities $\mathrm{d} F / \mathrm{d} T$ and any related quantity will be context specific whilst $G$ will be a stationary property of a linear dynamic system.

\subsubsection{Quantifying the relationship between feedback amplitudes and time constants}

The objective is to find the values of $g(\tau)$ that provide the least squares fit between the climate model $\Delta T$ and the output of Eqs. (1-3). To estimate $g(\tau)$ from these time series we start by pre-specifying values of $\tau$ that span the dynamic range of the model response we are investigating. If we are interested in capturing the full dynamic range of each climate model it is critical that we analyse perturbations taken through to full equilibrium. Figure $2 b$ shows the full equilibrium response of $\Delta T$ to the $4 \times \mathrm{CO}_{2}$ forcing shown in Fig. 2a for the three models being investigated. From this we can see that the temperature response is equilibrating in $<5000 \mathrm{yr}$. Therefore, if we specify $\tau=0,10,20, \ldots, 4990,5000 \mathrm{yr}$ this guarantees coverage of the dynamic response of the model because clearly the $5000 \mathrm{yr}$ time constant feedback cannot be contributing significantly in these cases. As we will see, $\tau<1000 \mathrm{yr}$ will actually be sufficient for the three models analysed here because feedback considerably extends the effective timescale of the response of $\Delta T$. A $10 \mathrm{yr}$ increment for $\tau$ was found to be an adequate trade off between, on the one hand capturing the subtleties of the $g(\tau)$ relationship, whilst on the other being coarse enough to make the estimation of $g(\tau)$ manageable.

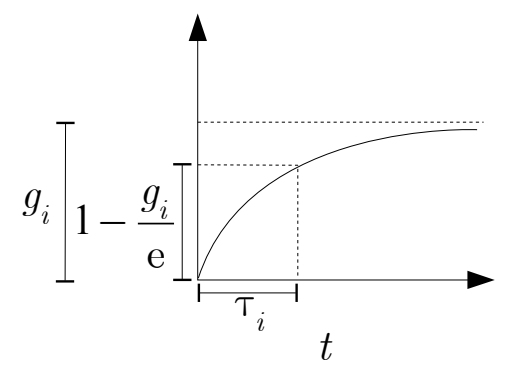

a.

b.

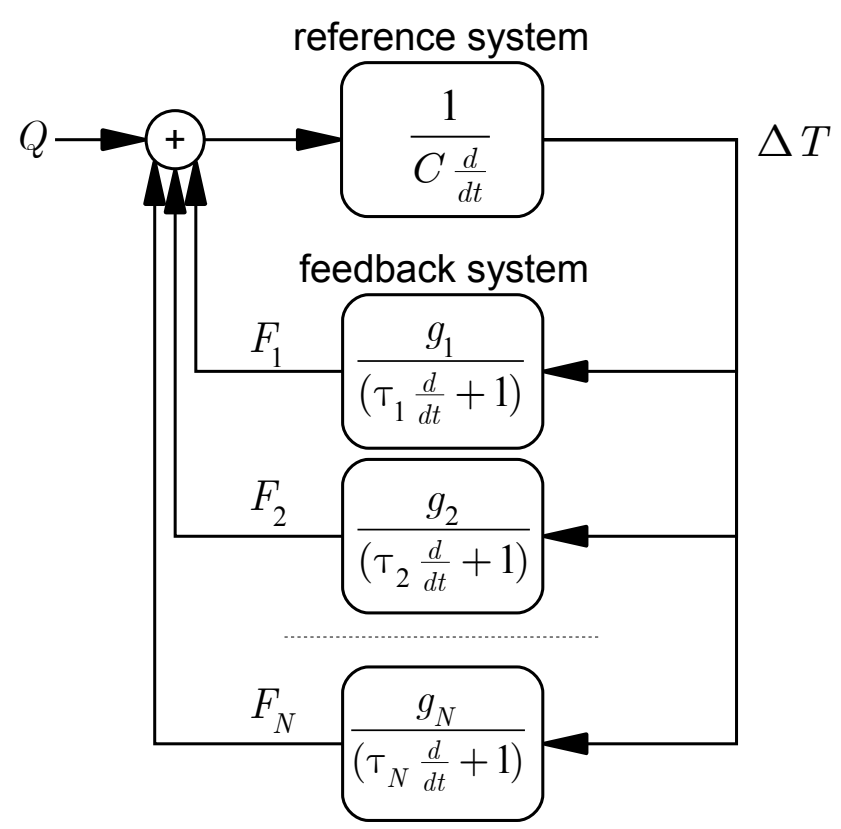

Fig. 1. (a) The generic response of the first order feedback elements shown in (b), showing the time constant $\tau$ in relation to its feedback amplitude $g$.

Having specified $\tau$ the aim is to estimate the corresponding values of $g$. Despite being a quasi-linear problem (sum of linear feedback amplitudes) it is also heavily over parameterised $(N=100)$ making direct search methods such as gradient descents impracticable. Furthermore, the dynamic behaviour of a large collection of first order responses as in Eq. (3) can often be represented exactly by a smaller subset of first order responses (Jarvis and Li, 2010; see later). Because of this, direct search methods will also tend to gravitate on low order approximations for $g(\tau)$ and not on the true relationship (Jarvis and Li, 2010). To avoid this we have elected to use slow cool simulated annealing to search for $g(\tau)$ because, not only is it more likely to cope with a high dimension search such as this, its inability to exploit any covariance between the individual estimates of $g$ means it will tend to avoid gravitating on low order approximations of the feedback dynamics. 

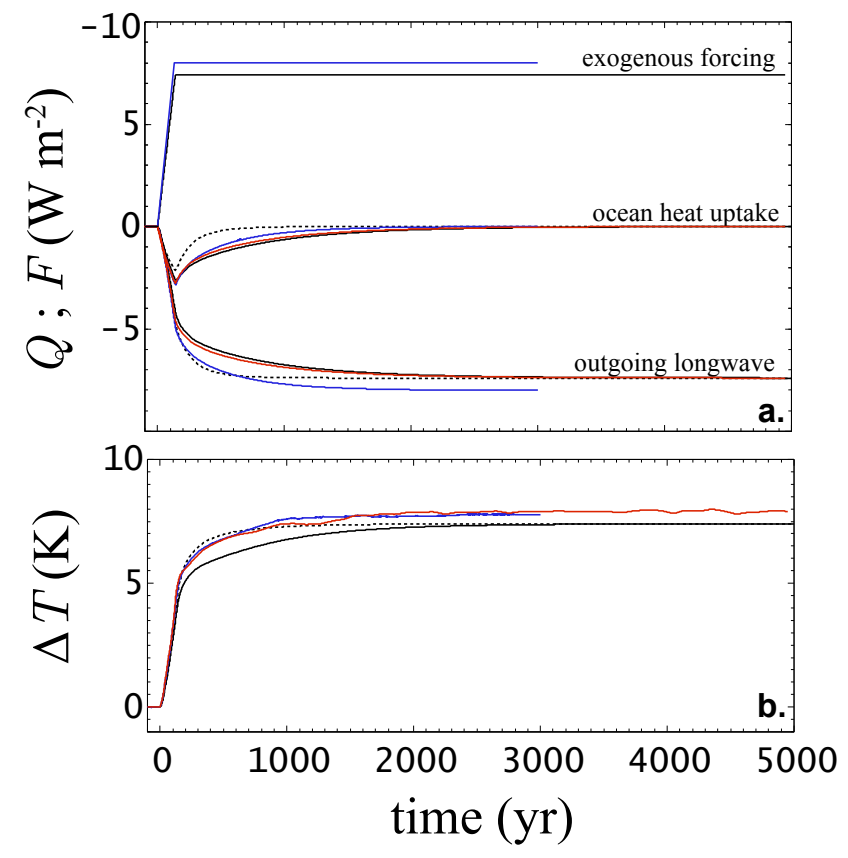

Fig. 2. (a) The exogenous forcings, ocean heat uptake and outgoing long wave radiative heat fluxes associated with the temperature perturbations shown in (b) (see text). The exogenous forcings marked black are common to both the GEBM and AOGCM model runs. (b) The global surface temperature response of three climate models to the exogenous forcings shown in (a): MAGICC-GEBM with (black) and without (black-dashed) polar sinking; UVicESCMEMIC (blue); GFDLR15a-AOGCM (red).

A finite difference approximation is used to vectorise Eqs. (1 to 3 ) so that the annealing code runs fast enough to be of utility. For this the reference system is given by,

$\Delta T(t)=\Delta T(t-\Delta t)+\frac{\Delta t}{C}[Q(t)+F(t-\Delta t)]$

and the feedback forcings are given by,

$F(t)=\sum_{i=1}^{N}\left[a_{i} F(t-\Delta t)+b_{i} \Delta T(t-\Delta t)\right]-3.3 \Delta T(t-\Delta t)$

where $g_{i}=b_{i} /\left(1-a_{i}\right)$ and $\tau_{i}=-\Delta t / \ln \left(a_{i}\right)$. The additional $\tau=0$ Planck feedback of $-3.3 \mathrm{~W} \mathrm{~m}^{-2} \mathrm{~K}^{-1}$, which captures the effects of temperature driven infrared heat loss (Forster and Taylor, 2006), is stated explicitly because, in addition to being relatively well defined, it provides a useful baseline against which all values of $g_{i}$ are estimated. All results presented for $G(\tau)$ will include this feedback. The annual sample interval, $\Delta t$, was found to be sufficient to avoid introducing significant numerical errors into the finite difference approximation.

The annealing conditions found to be appropriate for the estimation were $10^{6}$ iterations and a cooling rate of $10^{-6} \mathrm{~K}$ per iteration. Because each annealing result has embedded in it some of the random perturbations of the cooling process

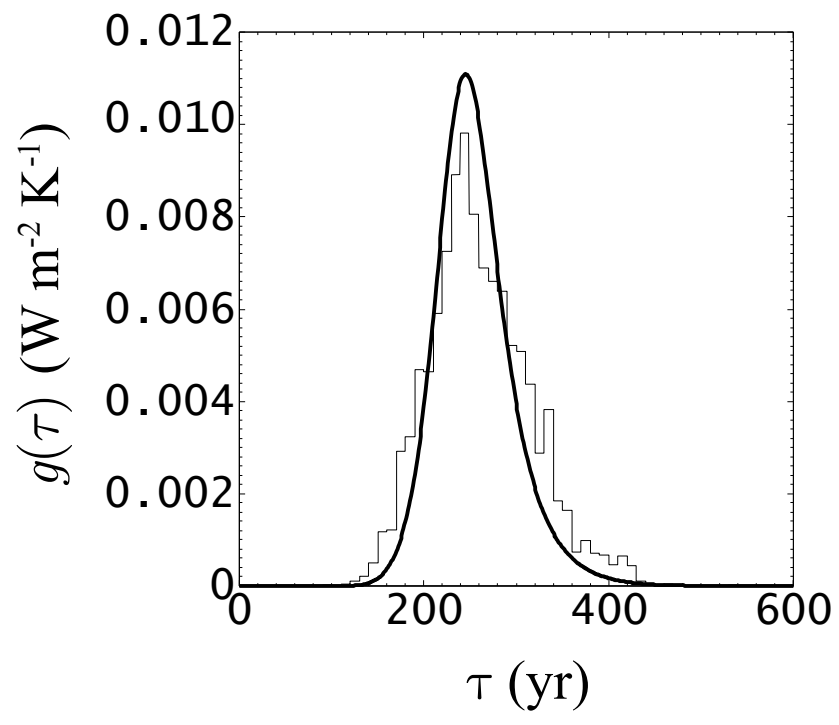

Fig. 3. The relationship between feedback amplitude, $g$, and its corresponding time constant, $\tau$, for a synthetic test case. The thick line has been used to generate a unit step response to which the structure in Fig. 1 has been optimised using the simulated annealing methodology outlined in the text. The thin line is the resultant estimate of the same relationship.

the results we present are the average of 100 individual anneals. This ensemble was also used as an approximate measure of the uncertainty in the $g(\tau)$ estimates. The estimation was done using the annealing algorithm in Yang et al. (2005) run in Matlab ${ }^{\mathrm{TM}} 7.0$, which on a $2.83 \mathrm{GHz}$ Intel Core 2 Quad machine took approximately $20 \mathrm{~h}$ for each 100 member ensemble. These conditions were found to provide reasonable results when recovering know synthetic $g(\tau)$ relationships, an example of which is shown in Fig. 3. It is clear from this test that there is a tendency for the method to partially smooth the estimate of $g(\tau)$ making it less peaked than it should be. This needs to be kept in mind when interpreting the results.

\subsubsection{Climate models and $\mathrm{CO}_{2}$ perturbation runs}

Fully equilibrated A-OGCM perturbation experiments are rare because of the expense of running such models for the necessary amount of time. As a result, a comprehensive intercomparison of A-OGCM model dynamics is not impossible at present, although as and when these data become available then clearly such an analysis could prove informative. In the meantime, this paper will look at one particular A-OGCM experiment; GFDL R15a perturbed using a $1 \%$ annual compound $\mathrm{CO}_{2}$ increase to $4 \times \mathrm{CO}_{2}$ (taking $140 \mathrm{yr}$ ) and then run to equilibrium (Manabe et al., 1991). To complement this, an equivalent analysis will be made of a GEBM (MAGICC as detailed in Eickout et al., 2004); and an EMIC (UVicESCM; Weaver et al., 2001) perturbed in exactly the same way. The three perturbation data sets are given in Fig. 2. 
Although these three models differ in the level of detail with which the climate system is represented, they share structural similarities, particularly in the representation of ocean heat uptake which, for all three, is comprised of both diffusive heat uptake across the thermocline and circulatory heat uptake via Meridional Overturning Circulation (MOC).

\subsection{Results and discussion}

\subsubsection{The estimated relationships for $g(\tau)$ and $G(\tau)$}

Figure 4 shows the relationships for the incremental feedback amplitudes, $g(\tau)$, and cumulative feedback amplitude, $G(\tau)$, estimated for the three models. Having accounted for the Planck feedback, the values of $g(\tau)$ are positive (Fig. 4a) and, as a result, $G(\tau)$ becomes progressively less negative with increasing $\tau$ (Fig. 4b). $g(\tau)$ is at a maximum when $\tau=0$, highlighting the importance of positive feedbacks in modulating the Planck feedback on the sub-annual timescale in these models (Bony et al., 2006; Forster and Gregory, 2006; Soden and Held, 2006). For $\tau>0, g(\tau)$ remains positive but tends to zero. The only process that is common to all three models that can explain this is the loss of ocean heat uptake from the surface as the oceans accumulate heat when equilibrating with surface temperature. Under the assumption that $g(\tau)$ for $\tau>0$ are attributable to this effect alone and, as $\tau \rightarrow \infty, g_{0}(\tau) \rightarrow 0$ where $\mathrm{O}$ denotes "ocean" feedbacks, then,

$g_{0}(0)=\sum_{\tau>0}^{\infty} g(\tau)$

and,

$G_{\mathrm{o}}(\tau)=\sum_{\tau>0}^{\tau} g(\tau)$

The inset in Fig. 4b shows how $G_{0}(\tau)$ dissipates with increasing $\tau$ as a consequence of the ocean heat equilibration. Baker and Roe (2009) also treated the ocean heat equilibration process as a transient negative feedback in a simple GEBM (see their Fig. 6b), although they use time as a measure of timescale rather than time constants. When viewed as a function of time constant (i.e. Fig. 4), a transient negative feedback can be understood as a negative feedback that is eroded by a series of lagged positive feedbacks that represent the progressive loss of the ocean heat sink.

For all three models $g_{0}(0) \approx-1.5 \mathrm{~W} \mathrm{~m}^{-2} \mathrm{~K}^{-1}$ (see Table 1), which is approximately twice the equivalent ocean feedback parameter estimate of $-0.6 \mathrm{~W} \mathrm{~m}^{-2} \mathrm{~K}^{-1}$ presented by Gregory and Forster (2008) for the CMIP4 A-OGCM ensemble. The reason for this is provided in Fig. $4 \mathrm{~b}$ which shows that, because the ocean heat feedback rapidly diminishes with increasing timescale, particularly for $\tau<200 \mathrm{yr}$, the feedback amplitude estimate will be heavily dependent on the degree of equilibration in the data used in the estimation. As with the estimates of Raper et al. (2002), Gregory
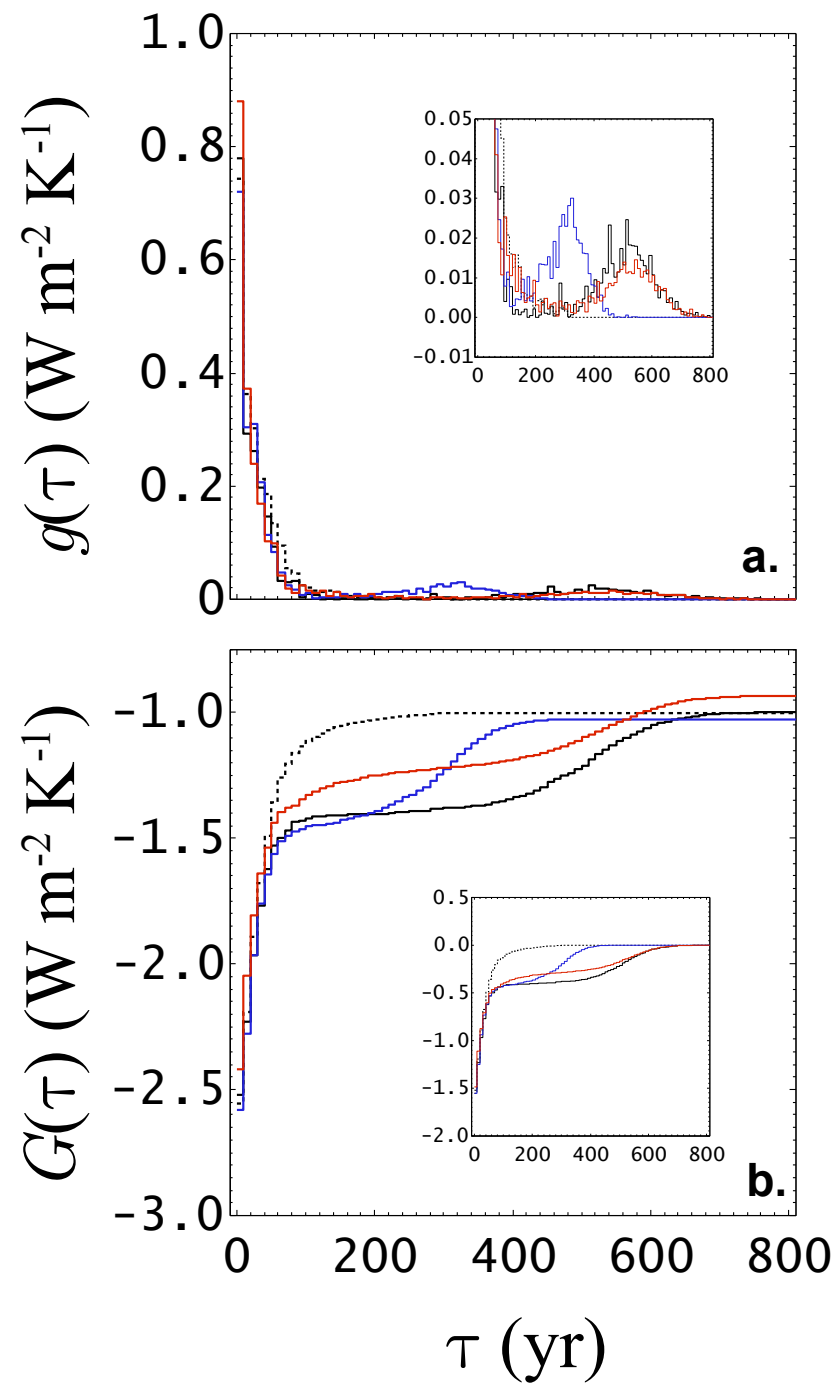

Fig. 4. (a) The relationship between feedback amplitude $g$ and time constant $\tau$ estimated from the data shown in Fig. 2: MAGICCGEBM with (black) and without (black-dashed) polar sinking; UVicESCM-EMIC (blue); GFDLR15a-AOGCM (red). The inset shows the tail of the relationship in more detail. (b) The relationship between the cumulative feedback amplitude $G$ and $\tau$. Here $G(\tau)=\sum_{\tau=0}^{\tau} g(\tau)-3.3$. The inset shows the cumulative ocean heat uptake feedback amplitude $G_{\mathrm{O}}$ (see text).

and Forster (2008) estimated the ocean heat uptake feedback amplitude from the first $70 \mathrm{yr}$ of the one percent compound $\mathrm{CO}_{2}$ forcing experiments. Under these circumstances one would estimate something in the region of $G_{0}(70 / \mathrm{e})$ which, from Fig. $4 \mathrm{~b}$, we can see is close to $-0.6 \mathrm{~W} \mathrm{~m}^{-2} \mathrm{~K}^{-1}$. This highlights the importance of correctly handling the dynamics in both real and model data when estimating dynamic feedback properties.

The rapid reductions in $g(\tau)$ for $\tau<200 \mathrm{yr}$ seen in Fig. 4 are indicative of the equilibration of a diffusion driven 
Table 1. Estimates of various parameters derived from the relationships shown Fig. 4 (see text for explanation). The values of $\tau_{\mathrm{d}, \mathrm{c}}$ are the median values of the distributions shown in Fig. $3 \mathrm{a}$ and $g_{\mathrm{d}, \mathrm{c}}$ are the areas under the distribution function. Note, $-g_{\mathrm{o}}(0)=g_{\mathrm{d}}+g_{\mathrm{c}}$. The 95 percent confidence intervals are derived from the 100 member ensemble of the simulated annealing results.

\begin{tabular}{|c|c|c|c|c|c|c|c|c|}
\hline Model & $\begin{array}{l}C, h \\
\left(\mathrm{~W} \mathrm{~m}{ }^{-2} \mathrm{~K}^{-1} \mathrm{a}^{-1}\right) \\
(\mathrm{m})\end{array}$ & $\begin{array}{l}\text { 5th, 95th } \\
\text { percentiles }\end{array}$ & 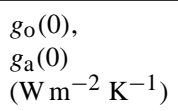 & $\begin{array}{l}5 \text { th, } 95 \text { th } \\
\text { percentiles }\end{array}$ & $\begin{array}{l}\tau_{\mathrm{r}}, \tau_{\mathrm{d}}, \tau_{\mathrm{c}} \\
(\mathrm{yr})\end{array}$ & $\begin{array}{l}5 \text { th, } 95 \text { th } \\
\text { percentiles }\end{array}$ & $\begin{array}{l}g_{\mathrm{r}}, g_{\mathrm{d}}, g_{\mathrm{c}} \\
\left(\mathrm{W} \mathrm{m}{ }^{-2} \mathrm{~K}^{-1}\right)\end{array}$ & $\begin{array}{l}5 \text { th, } 95 \text { th } \\
\text { percentiles }\end{array}$ \\
\hline $\begin{array}{l}\text { MAGICC } \\
(\pi=0.4)\end{array}$ & $\begin{array}{l}21.93 \\
168.95\end{array}$ & $\begin{array}{l}10.57,32.91 \\
81.43,253.54\end{array}$ & $\begin{array}{l}-1.52 \\
2.30\end{array}$ & $\begin{array}{l}-0.94,-2.09 \\
1.73,2.89\end{array}$ & $\begin{array}{l}8.70 \\
20 \\
500\end{array}$ & $\begin{array}{l}3.57,16.69 \\
- \\
-\end{array}$ & $\begin{array}{l}-2.52 \\
1.13 \\
0.39\end{array}$ & $\begin{array}{l}-3.09,-1.94 \\
0.50,1.71 \\
0.29,0.45\end{array}$ \\
\hline $\begin{array}{l}\text { MAGICC } \\
(\pi=0.0)\end{array}$ & $\begin{array}{l}18.67 \\
143.83\end{array}$ & $\begin{array}{l}9.60,27.48 \\
73.96,211.71\end{array}$ & $\begin{array}{l}-1.55 \\
2.30\end{array}$ & $\begin{array}{l}-1.03,-2.05 \\
1.812 .83\end{array}$ & - & - & - & - \\
\hline UVicESCM & $\begin{array}{l}9.59 \\
73.88\end{array}$ & $\begin{array}{l}2.22,17.42 \\
17.10,134.20\end{array}$ & $\begin{array}{l}-1.55 \\
2.27\end{array}$ & $\begin{array}{l}-1.08,-2.04 \\
1.79,2.74\end{array}$ & $\begin{array}{l}3.72 \\
20 \\
300\end{array}$ & $\begin{array}{l}0.75-8.26 \\
- \\
-\end{array}$ & $\begin{array}{l}-2.58 \\
1.14 \\
0.41\end{array}$ & $\begin{array}{l}-3.06,-2.11 \\
0.60,1.63 \\
0.33,0.51\end{array}$ \\
\hline GFDLR15a & $\begin{array}{l}4.35 \\
33.51\end{array}$ & $\begin{array}{l}1.67,9.28 \\
12.87,71.49\end{array}$ & $\begin{array}{l}-1.49 \\
2.37\end{array}$ & $\begin{array}{l}-1.06,-2.01 \\
1.84,2.79\end{array}$ & $\begin{array}{l}1.80 \\
15 \\
485\end{array}$ & $\begin{array}{l}0.58,4.58 \\
- \\
-\end{array}$ & $\begin{array}{l}-2.42 \\
1.19 \\
0.30\end{array}$ & $\begin{array}{l}-2.94,-2.00 \\
0.78,1.69 \\
0.22-0.35\end{array}$ \\
\hline
\end{tabular}

mechanism where $g \propto \tau^{-2}$ (Bowman, 1995). This process is common to all three models through the diffusive component of ocean heat uptake. Figure 4 also shows that, after the initial stabilisation of $G(\tau)$ as $g(\tau) \rightarrow 0$ at $\tau \approx 200 \mathrm{yr}$, all three models reveal further increases thereafter, i.e. a secondary set of positive feedbacks are experienced (see Fig. 4a inset). This behaviour was identified by Jarvis and $\mathrm{Li}$ (2010) for a range of climate models looking at the feedforward ${ }^{1}$ dynamics of the mean surface temperature response. The cause for this behaviour can be demonstrated using the GEBM model. Like the more complex EMIC and A-OGCM, in this model surface heat is transferred to the oceans through both diffusion into the near surface ocean across the thermocline, and through large scale Meridional Overturning Circulation (MOC) driving heat directly into the deep ocean from the surface (Hoffert et al., 1980). If we switch off the MOC pathway by setting the polar sinking term to zero in the GEBM we see that this abolishes the secondary long timescale peak in $g(\tau)$ (see Fig. 4a) and the remaining purely diffusive ocean heat uptake reverts to $g \propto \tau^{-2}$ as expected (see Fig. 4a). From this result the bi-modal relationship for $g(\tau)$ that is observed for the EMIC and the A-OGCM is understandable. The diffusive ocean heat uptake regime of the thermocline in these models gives rise to the inverse component of $g(\tau)$ for $\tau<200 \mathrm{yr}$. The MOC heat uptake gives rise to the secondary peaked distribution in $g(\tau)$ for $\tau>200 \mathrm{yr}$. The reason this secondary component of $g(\tau)$ is peaked and not $g \propto \tau^{-2}$ is because, unlike diffusion, circulatory heat distribution is a mixing process. Well mixed systems are characterised by a single time constant and feedback amplitude i.e. they are infinitely peaked in $g(\tau)$. Rather than being infinitely peaked,

\footnotetext{
${ }^{1}$ As the name implies, feedforward is the opposite to feedback i.e. it is the dynamic associated with passing the input forward to the output.
}

partially mixed systems such as the global intermediate and deep oceans, will be characterised by a distribution of time constants and amplitudes about a peak dependent of the degree of mixing. As the system tends more toward a purely diffusive regime so $g(\tau)$ will tend toward $g \propto \tau^{-2}$.

An alternative but related way of accounting for the $g(\tau)$ distribution of the deep ocean is to consider the surface as being linked to a very large number of deep ocean water bodies. Each of these is connected to the surface in different ways depending on the circulatory architecture of the ocean. This is equivalent to there being a distribution of values of $g$ and $\tau$ to describe the connectivity between these water bodies and the surface, with the first moment of this distribution describing the most common degree of connectivity. It is important to keep in mind that the annealing method does have a tendency to smooth the peak in $g(\tau)$ (see Fig. 3) and, therefore, it is likely to be slightly more peaked than shown in Fig. 4.

For all three models these long timescale adjustments to the oceanic feedback amount to the preservation of a feedback amplitude of approximately $-0.35 \mathrm{~W} \mathrm{~m}^{-2} \mathrm{~K}^{-1}$ up until the equilibration of this process. We see from Fig. 4 that this secondary equilibration starts at around $\tau \approx 200 \mathrm{yr}$ and finishes at $\tau \approx 400 \mathrm{yr}$ for the EMIC, whilst for the A-OGCM model this process occurs some 200 yr later. Stouffer (2004) plots the spatial distribution of the times taken to achieve either 30 or 70 percent equilibration of ocean temperature in the same A-OGCM, but run under $2 \times \mathrm{CO}_{2}$ forcing. The 30 percent equilibration ranges from 100 to $900 \mathrm{yr}$ whilst the 70 percent timescale ranges from 100 to $1800 \mathrm{yr}$. It would be tempting to try and relate these spatial distributions to the results shown in Fig. 4. However, it is not clear at this stage how to map Stouffer's (2004) results into feedbacks experienced at the surface and hence $g(\tau)$. 
Because $G(0)=-3.3+g_{\mathrm{o}}(0)+g_{\mathrm{a}}(0)$, where $g_{\mathrm{a}}(0)$ is the instantaneous net atmospheric feedback amplitude, it is possible to estimate $g_{\mathrm{a}}(0)$ because $g_{\mathrm{o}}(0)$ is known. The results for the three models are given in Table 1 and show relatively little difference between the three with $g_{\mathrm{a}}(0) \sim-2.3 \mathrm{~W} \mathrm{~m}^{-2} \mathrm{~K}^{-1}$. This estimate is indistinguishable from the estimate of $2.2 \pm 0.26 \mathrm{~W} \mathrm{~m}^{-2} \mathrm{~K}^{-1}$ for the same quantity made by Colman (2003) for a suite of A-OGCM's by summing individual estimates of the water vapour, cloud, albedo, and lapse rate feedback parameters. This again provides a degree of independent verification of the annealing results. The GEBM has no explicit representation for $g_{\mathrm{a}}(0)$ and hence its behaviour in this regard is a result of an implicit parameterization. This is not surprising given GEBM's including MAGICC are often tuned to emulate AOGCM's (Raper et al., 2002). For reference, Fig. 2b shows the outgoing longwave forcing $F_{\mathrm{a}}=\left(-3.3+g_{\mathrm{a}}(0)\right) \Delta T$ for all three models.

Table 1 also shows the estimated values of the surface heat capacity $C$ and equivalent $1 \mathrm{~d}$ well-mixed ocean depths of the three models. This is significantly larger in the GEBM due to having a parameterisation for ocean heat uptake that allows for the effective downward penetration of the mixed layer into the thermocline with time (Hansen et al., 1985; Watterson, 2000), meaning this extends well beyond the $90 \mathrm{~m} \mathrm{spec}-$ ified in this model. In contrast, the surface heat capacity of the EMIC and AOGCM are much closer to that expected for the real world surface mixed layer with equivalent depths of 74 and $34 \mathrm{~m}$ respectively, implying a somewhat more realistic representation of the coupling between the surface and lower ocean.

\subsubsection{Implications for simple models}

The results in Fig. 4a suggest highly reduced order forms of these climate models can be identified which summarise of the core dynamic characteristics of surface energy balance. This reduction in model order stems from the fact that, providing they are either low variance or symmetric, the dynamics produced by distributions of first order processes such as those shown in Fig. 4a can be accurately represented by the first moment of these distributions (Jarvis and $\mathrm{Li}, 2010$ ). Given we have two such distributions then, taking into account the reference system, a reduced third order model is implied. Third order analogues of the climate system energy balance have been proposed previously (e.g. Dickinson, 1981; Greiser and Schonweis, 2001; Li and Jarvis, 2009). However, here we will define the structure and parameterisation directly from the feedback analysis results.

Figure 4 suggests one atmospheric and two transient ocean heat feedbacks operating on the reference system i.e.,

$C \frac{\mathrm{d}}{\mathrm{d} t} \Delta T=Q-F_{\mathrm{a}}-F_{\mathrm{d}}-F_{\mathrm{c}}$

where $F_{\mathrm{a}}=\left(3.3-g_{\mathrm{a}}\right) \Delta T$ is the atmospheric feedback forcing; $F_{\mathrm{d}}=g_{\mathrm{d}}\left(\Delta T-\Delta T_{\mathrm{d}}\right)$ is the diffusive ocean heat uptake and $F_{\mathrm{c}}=g_{\mathrm{c}}\left(\Delta T-\Delta T_{\mathrm{c}}\right)$ is the circulatory ocean heat uptake. This structure differs from the usual layer cascade box models (Dickinson, 1981; Greiser and Schonweis, 2001; Hooss, 2001; Li and Jarvis, 2009) because the two ocean heat reservoirs interact directly with the ocean mixed layer but not each other. $g_{\mathrm{d}, \mathrm{c}}$ are both the ocean heat exchange efficiencies and the feedback amplitudes of these two processes. From this we get,

$C \frac{\mathrm{d}}{\mathrm{d} t} \Delta T(t)=Q(t)+g_{\mathrm{r}} \Delta T(t)+g_{\mathrm{d}} \Delta T_{\mathrm{d}}(t)+g_{\mathrm{c}} \Delta T_{\mathrm{c}}(t)$

$\tau_{\mathrm{d}, \mathrm{c}} \frac{\mathrm{d}}{\mathrm{d} t} \Delta T_{\mathrm{d}, \mathrm{c}}(t)=g_{\mathrm{d}, \mathrm{c}} \Delta T(t)-\Delta T_{\mathrm{d}, \mathrm{c}}(t)$

where $g_{\mathrm{r}}=-3.3+g_{\mathrm{a}}-g_{\mathrm{d}}-g_{\mathrm{c}}$ i.e. all $\tau=0$ feedback terms aggregate in the reference system. It is tempting to formulate Eq. (13) in the form of Eq. (12) by specifying heat capacities for the two oceanic responses. We will avoid this because, as with the mixed layer, this would imply the temperature states $\Delta T_{\mathrm{d}, \mathrm{c}}$ represent well mixed conditions. Clearly this is not so and, were we to do this, the implied heat capacities would be significantly less than that known to reside in the parent models (Grieser and Schönwiese, 2001). Instead, we have retained $\tau_{\mathrm{d}, \mathrm{c}}$ to describe the average dynamic timescales over which the ocean heat equilibrates.

The parameters for Eq. (10) for the three climate models are given in Table 1 . In each case the reduced order models are able to account for more than 99.9 percent of the variance of their higher order parents. The values of $\tau_{\mathrm{d}}$ are comparable amongst the three models whereas the value of $\tau_{\mathrm{c}}$ is significantly smaller for the EMIC. The values of $g_{\mathrm{d}, \mathrm{c}}$ do not depend on the structure of Eq. (10) because they are simply the areas under the two distributions shown in Fig. 4a for $\tau>0$. As a result, these values should be representative of the actual efficiencies of heat exchange with the diffusive and circulatory ocean regimes in these models. The values of $g_{\mathrm{d}, \mathrm{c}}$ shown in Table 1 are comparable between the three models, although $g_{\mathrm{c}}$ is smaller in the A-OGCM consistent with the finding that the $4 \times \mathrm{CO}_{2}$ forcing of this model was associated with significant weakening of MOC (Manabe et al., 1991). The ratio $g_{\mathrm{d}, \mathrm{c}} /\left(g_{\mathrm{d}}+g_{\mathrm{c}}\right)$ is a measure of the relative importance of the two ocean heat uptake pathways in the ocean heat uptake feedback. From the values in Table 1 this partitioning is approximately $3: 1$, diffusive:circulatory for the GEBM and EMIC models and 4:1 for the AOGCM, demonstrating the decadal timescale diffusive heat loss pathway is significantly more important in affecting the reference system energy balance dynamics in these three models. Because the MOC heat uptake pathway appears less important for determining the dynamics of the surface temperature response, whilst also being very uncertain, this suggests that dropping this feedback term from simple climate models would not radically impact on model performance, particularly for timescales $<200 \mathrm{yr}$. 


\section{Conclusions}

Such a small sample of climate models is not intended to reflect the diversity of climate model behaviour. This sample was determined by the desire to represent a spectrum of climate model types and the rarity of full equilibrium run AOGCM data sets. The analysis presented demonstrates how the magnitude of the feedbacks are related to their response timescale in these models. This relationship has three general traits. Firstly, it is comprised of a large net negative feedback at zero lag determined by outgoing long wave and ocean heat uptake effects which are partially offset by instantaneous atmospheric adjustment. As feedback response timescale increases this net negative feedback is attenuated by a series of positive feedbacks which I argue are attributable to the equilibration of heat between the surface and the oceans. This equilibration process appears to be divisible into two discrete components; a decadal timescale equilibration which relates to diffusive ocean heat uptake accounting for approximately 75 to 80 percent of the total equilibration dynamic; and a centuries timescale equilibration which relates to the large scale circulation of heat into the oceans accounting for the remaining 20 to 25 percent. To see if the results presented here are more general obviously requires more full equilibrium run data sets becoming available. Because ocean circulation can depend on the nature of the forcing applied to A-OGCM's (Manabe et al., 1991; Stouffer, 2004) $g(\tau)$ can be a function of the exogenous disturbance making any generalisation more difficult. It must be stressed that this is because the underlying dynamics of the A-OGCM are nonlinear or nonstationary and not because the estimates of the feedback amplitudes are biased by using a static analysis framework on a dynamic system (see e.g. Gregory et al., 2004).

The results from the feedback magnitude-timescale analysis highlight that the link between simple linear (box and impulse response) climate models and their complex counterparts is not as superficial as originally believed. This link is partly explained by the fact that imperfect mixing processes in the ocean can still have characteristic time constants and amplitudes, hence the peak in $g(\tau)$. Imperfect mixing is analogous to perfect mixing occurring within a fraction of the control volume (Beer and Young, 1983). As a result, the ratio of the actual control volume to that estimated for an appropriate box model analogue could provide a useful measure of the degree of ocean mixing in play. As with most box model analogues of the global energy balance, if $c_{\mathrm{d}, \mathrm{c}}=\tau_{\mathrm{d}, \mathrm{c}} / g_{\mathrm{d}, \mathrm{c}}$, then the parameter values in Table 1 give rise to approximately one quarter of the heat capacity known to reside in the global oceans.

Aires and Rossow (2003) rightly highlight a range of limitations of adopting a linear feedback approach for characterising feedback processes, and in particular the inappropriate accounting for interactions between feedbacks the linear additive framework provides. The linear systems approach need not be restricted to additive feedbacks and could also consider a range of configurations of first order systems which map to physical processes (see e.g. Li et al., 2009 for a global carbon cycle example). However, this would require relatively detailed knowledge of the 3-D architecture of the global energy balance system. This is why the analysis presented here was initially restricted to identifying the magnitude of feedbacks operating on particular timescales with the interpretation in terms of processes only coming in light of the results. Therefore, the main limitation of the current approach is not the additive structure of the feedbacks but the assumed time invariance of the feedback amplitudes. For example, it is clear from Manabe et al. (1991) that the $4 \times \mathrm{CO}_{2}$ A-OGCM run analysed here has non-stationary ocean circulation which would equate to a degree of nonstationarity in $g(\tau)$. That said, it is remarkable how well linear frameworks like this perform when accounting for A-OGCM behaviour (see also Grieser and Schönwiese, 2001; Gregory et al., 2004; Forster and Taylor, 2006; Li and Jarvis, 2009). Two candidate mechanisms for giving rise to this are the nature of the network connections within these models and the overall balance of negative over positive feedback. Further research on these traits is needed.

Acknowledgements. I would like to thank David Leedal, Paul Smith, Arun Chotai and Sile Li for valuable discussions had during the development of the analysis and Peter Young for ongoing discussions on climate model dynamics. I also express my gratitude to Michael Eby, Gokhan Danabasoglu and Ronald Stouffer who kindly provided the equilibrated climate model data. This paper has benefitted significantly from the comments of two anonymous reviewers and the editorial guidance of Piers Forster.

Edited by: V. Lucarini

\section{References}

Aires, F. and Rossow, W. B.: Inferring instantaneous, multivariate and nonlinear sensitivities for the analysis of feedback processes in a dynamical system: Lorenz model case-study, Q. J. Roy. Meteorol. Soc., 129, 239-275, 2003.

Baker, M. B. and Roe, G. H.: The shape of things to come: why is climate change so unpredictable?, J. Climate, 22, 4574-4589, 2009.

Bates, J. R.: Some considerations of the concept of climate feedback, Q. J. Roy. Meteorol. Soc., 133, 545-560, 2007.

Beer, T. and Young, P. C.: Longitudinal dispersion in natural streams, J. Environ. Eng., 109, 1049-1067, 1983.

Bony, S., Colman, R., Kattsov, V. M., Allan, R. P., Bretherton, C. S., Dufresne, J.-L., Hall, A., Hallegatte, S., Holland, M. M., Ingram, W., Randall, D. A., Soden, B. J., Tselioudis, G., and Webb, M. J.: How well do we understand and evaluate climate change feedback processes?, J. Climate, 19, 3445-3482, 2006.

Bowman, K. P.: Diffusive transport by breaking Waves, J. Atmos. Sci., 52, 2146-2427, 1995.

Colman, R. A.: A comparison of climate feedbacks in general circulation models, Clim. Dynam., 20, 865-873, 2003. 
Danabasoglu, G. and Gent, P. R.: Equilibrium climate sensitivity: is it accurate to use a slab ocean model?, J. Climate , 22, 24942499, 2009.

Dickinson, R. E.: Convergence Rate and Stability of OceanAtmosphere Coupling Schemes with a Zero-Dimensional Climate Model, J. Atmos. Sci., 38, 2112-2120, 1981.

Dickinson, R. E. and Schaudt, K. J.: Analysis of timescales of response of a simple climate model, J. Climate, 11, 97-106, 1998.

Eickhout, B., den Elzen, M. G. J., and Kreileman, G. J. J.: The Atmosphere-Ocean System of IMAGE 2.2. A global model approach for atmospheric concentrations, and climate and sea level projections. RIVM report 481508017/2004, RIVM, Bilthoven, 2004.

Forster, P. M. and Taylor, K. E.: Climate Forcings and Climate Sensitivities Diagnosed from Coupled Climate Model Integrations, J. Climate, 19, 6181-6194, 2006.

Gregory, J. M. and Forster, P. M.: Transient climate response estimated from radiative forcing and observed temperature change, J. Geophys. Res., 113, D23105, doi:10.1029/2008JD010405, 2008.

Gregory, J. M., Ingram, W. J., Palmer, M. A., Jones, G. S., Stott, P. A., Thorpe, R. B., Lowe, J. A., Johns, T. C., and Williams, K. D.: A new method for diagnosing radiative forcing and climate sensitivity, Geophys. Res. Lett. 31, L03205, doi:10.1029/2003GL018747, 2004.

Grieser, J. and Schönwiese, C.-D.: Process, forcing, and signal analysis of global mean temperature variations by means of a three-box energy balance model, Climatic Change, 48, 617-646, 2001.

Hallegatte, S., Lahellec, A., and Grandpeix, J. Y.: An elicitation of the dynamic nature of water vapor feedback in climate change using a 1D model, J. Atmos. Sci., 63, 1878-1894, 2006.

Hansen, J. E., Lacis, A., Rind, D., Russell, G., Stone, P., Fung, I., Ruedy, R., and Lerner, J.: Climate sensitivity: analysis of feedback mechanisms, in: Climate Processes and Climate Sensitivity, edited by: Hansen, J. E. and Takahashi, T., American Geophysical Union, Washington DC, USA, 130-163, 1984.

Hansen, J. E., Russell, G., Lacis, A., Fung, I., Rind, D., and Stone, P.: Climate response times: Dependence on climate sensitivity and ocean mixing, Science, 229, 857-859, 1985.

Hansen, J. E., Sato, M., Kharecha, P., Russell, G., Lea, D. W., and Siddall, M.: Climate change and trace gases, Philos. Trans. Roy. Soc. A, 365, 1925-1954, 2007.

Hoffert, M. I., Calligari, A. J., and Hseich, C.-T.: The role of deep sea heat storage in the secular response to climate forcing, J. Geophys. Res., 85, 6667-6679, 1980.

Hooss, G.: Aggregate models of climate change: developments and applications, PhD thesis, Max-Planck-Institute for Meteorology, Hamburg, Germany, 2001.

Jarvis, A. J. and Li, S.: On the timescale - gain relationship of climate models, Clim. Dynam., 36, 523-531, 2010.
Knutti, R. and Hegerl, G. C.: The equilibrium sensitivity of the Earth's temperature to radiation change, Nat. Geosci., 1, 735743, 2008.

Li, S. and Jarvis, A. J.: The global mean surface temperature dynamics of an AOGCM: The HadCM3 $4 \times \mathrm{CO}_{2}$ forcing experiment revisited, Clim. Dynam., 33, 817-825, 2009.

Li, S., Jarvis, A. J., and Leedal, D. T.: Are response function representations of the global carbon cycle ever interpretable?, Tellus, 61B, 361-371, 2009.

Lu, J. and Cai, M.: A new framework for isolating individual feedback processes in coupled general circulation climate models. Part I: formulation, Clim. Dynam., 32, 873-885, 2009.

Manabe, S., Stouffer, R. J., Spelman, M. J., and Bryan, K.: Transient responses of a coupled ocean-atmosphere model to gradual changes of atmospheric $\mathrm{CO}_{2}$. Part I. Annual mean response, J. Climate, 4, 785-817, 1991.

Prather, M. J.: Time scales in atmospheric chemistry: Theory, GWPs for $\mathrm{CH}_{4}$ and $\mathrm{CO}$, and runaway growth, Geophys. Res. Lett., 23, 2597-2600, 1996.

Raper, S. C. B., Gregory, J. M., and Stouffer, R. J.: The role of climate sensitivity and ocean heat uptake on AOGCM transient temperature response, J. Climate, 15, 124-130, 2002.

Roe, G. H.: Feedbacks, timescales and seeing red, Ann. Rev. Earth Planet. Sci., 37, 93-115, 2009.

Roe, G. H. and Baker, M. B.: Why is climate sensitivity so unpredictable?, Science, 318, 629-632, 2007.

Soden, B. J. and Held, I. M.: An assessment of climate feedbacks in coupled ocean-atmosphere models, J. Climate, 19, 3354-3360, 2006.

Stephens, G. L.: Cloud Feedbacks in the Climate System: A Critical Review, J. Climate, 18, 237-273, 2005.

Stouffer, R. J.: Time scales of climate response, J. Climate, 17, 209-217, 2004.

Watterson, I. G.: Interpretation of simulated global warming using a simple model, J. Climate, 13, 202-215, 2000.

Watts, R. G., Morantine, M. C., and Achutarao, K.: Timescales in energy balance climate models. 1. The limiting case solutions, J. Geophys. Res., 99, 3631-3641, 1994.

Weaver, A. J.: The UVic Earth System Climate Model: Model description, climatology and application to past, present and future climates, Atmosphere Ocean, 39, 361-428, 2001.

Wigley, T. M. L. and Schlesinger, M. E.: Analytical solution for the effect on increasing $\mathrm{CO}_{2}$ on global mean temperature, Nature, 315, 649-652, 1985.

Williams, K. D., Ingram, W. J., and Gregory, J. M.: Time variation of effective climate sensitivity in GCMs, J. Climate, 2119, 50765090, 2008.

Yang, W. Y., Cao, W., Chung, T.-S., and Morris, J.: Applied numerical method using MATLAB, Wiley-Interscience, New Jersey, 2005. 